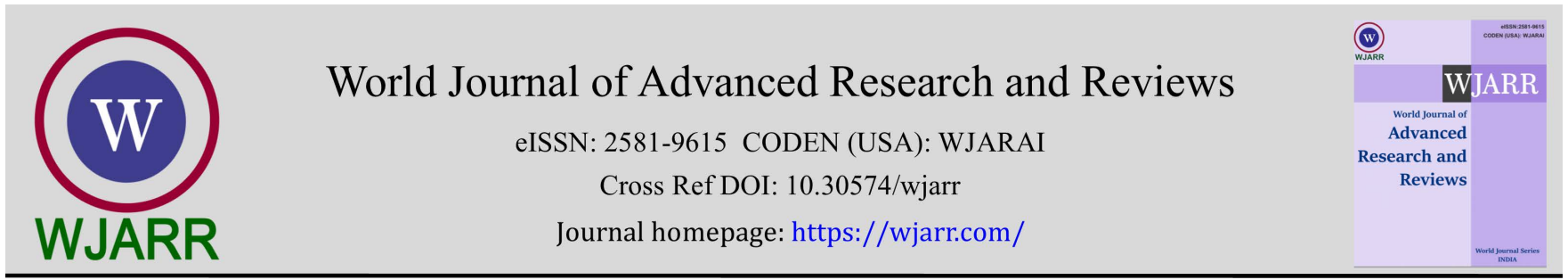

(RESEARCH ARTiClE)

Check for updates

\title{
Assessment of Severity of Liver Cirrhosis and Nutritional status of Cirrhotic patients in Tribhuvan University Teaching Hospital, Kathmandu
}

\author{
Yogita Sapkota ${ }^{1,}{ }^{*}$, Arun Gnawali ${ }^{2}$, Kalpana Tiwari ${ }^{3}$, Tulsi Maya Sharma ${ }^{1}$ and Basanti Thapa ${ }^{1}$ \\ ${ }^{1}$ Department of Nutrition and Dietetics, Faculty of Science and Technology, Purbanchal University, College of applied Food \\ and Dairy Technology (CAFODAT), Kumaripati, Lalitpur, Nepal. \\ ${ }^{2}$ Department of Gastroenterology, Tribhuvan University Teaching Hospital, Maharajgunj, Kathmandu, Nepal. \\ ${ }^{3}$ Department of Research and Planning, Faculty of Science and Technology, Purbanchal University, College of applied Food \\ and Dairy Technology (CAFODAT), Kumaripati, Lalitpur, Nepal.
}

World Journal of Advanced Research and Reviews, 2022, 13(02), 239-251

Publication history: Received on 07 January 2022; revised on 09 February 2022; accepted on 11 February 2022

Article DOI: https://doi.org/10.30574/wjarr.2022.13.2.0145

\begin{abstract}
Malnutrition is common in chronic liver disease. The general objective of this study was to assess severity and nutritional status of cirrhotic patients in Tribhuvan University Teaching Hospital (TUTH). A cross-sectional study was carried out among 88 cirrhotic patients of age group of $\geq 18$ years. Information regarding general profile of patient, Child Turcotte Pugh score (CTP), Model for End Stage Liver Diseases-Na Score ( MELD-Na), Subjective Global Assessment (SGA), Mid Arm Circumference (MAC), Triceps Skin Fold Thickness (TST), Mid Arm Muscle Circumference (MAMC) and Functional Assessment: Handgrip Strength (HGS) was collected. SPSS version 20 was used to analyse data.

Majority (56.8\%) belonged to CTP grade C, 31.8\% belonged to CTP grade B and $11.4 \%$ patients belonged to CTP grade A. Majority, $72.7 \%, 71.6 \%$ and $65.9 \%$ were malnourished when assessed using SGA, TST and MAC respectively. Majority, $86.4 \%$ of the patients had impaired handgrip strength. SGA, TST, MAC and HGS were significantly associated with CTP whereas no any statistically significant association was found between Child-Pugh and MAMC. There was statistically significant difference among the MELD Na means among SGA and HGS classes. The mean MELD Na scores did not significantly differ between among the TST, MAC and MAMC classes. The early diagnosis of the nutritional state and the treatment of malnutrition in cirrhotic patients can contribute to a reduction in the frequency and/or severity of these complications. SGA, TST, MAC and HGS are the non-invasive and easy methods of nutritional assessment of cirrhotic patients to use in regular clinical practice at bed side.
\end{abstract}

Keywords: Malnutrition; Chronic liver diseases; Severity; Nutritional status

\section{Introduction}

Malnutrition is common in chronic liver disease. Malnutrition appears to contribute to many of the complications of cirrhosis but it is difficult to assess the independent effect since progressive liver insufficiency is usually associated with both declining nutritional status and increased complications [1]. It is unclear whether malnourished cirrhotics are more likely to die as a result of compromised nutritional status or whether patients who die are simply more likely to be malnourished. Whatever the cause of the relationship, poor nutritional status is associated with very poor outcomes like increased infection rates, long stay in the intensive care unit and overall hospital stay and more use of blood products [2].

\footnotetext{
* Corresponding author: Yogita Sapkota

Department of Nutrition and Dietetics, Faculty of Science and Technology, Purbanchal University, College of applied Food and Dairy Technology(CAFODAT), Kumaripati, Lalitpur, Nepal.
}

Copyright (C) 2022 Author(s) retain the copyright of this article. This article is published under the terms of the Creative Commons Attribution Liscense 4.0. 
Malnutrition in all forms of liver disease is often under recognized and under treated despite the fact that appropriate treatment can improve outcomes. Although signs of malnutrition may be obvious in many liver patients, nutritional risk can be easily overlooked, and a high index of clinical suspicion is always appropriate [3].

Liver cirrhosis is a common disease in Nepal. However national data on the exact burden of diseases and studies on relationship with cirrhosis and nutrition are lacking [4]. Little attention is paid in evaluating nutrition in this group of patients [5]. The worldwide prevalence of malnutrition among cirrhotic patients ranges from 50 to $99 \%$ depending upon the different assessment methods [6] and also due to variation and lack of validated tools. Previously used parameters to assess nutritional status did not perform well in patients with cirrhosis. Various factors make traditional measurements imprecise for assessing the nutritional status. Malnutrition is also associated with the severity of the disease among hospitalized patients with cirrhosis and other complications such as ascites and bacterial infections. Although the pathogenesis of malnutrition is multifactorial and leads to a poor prognosis for the liver patient, it is commonly undiagnosed due to the complications of liver disease such as edema and ascites, which make weight change detection more difficult in this patient population [7]. Even if malnutrition is diagnosed in a patient, its importance is often underestimated by the physician and it is not considered a medical problem in need of immediate attention.

As of now, there are no specific guidelines for nutritional assessment in these patients. However, it is recommended that all patients with liver cirrhosis undergo a rapid nutritional assessment test to determine the risk of malnutrition. Those who are at risk should then be subjected to a more detailed nutritional assessment to confirm the presence and severity of malnutrition. Despite a correlation between poor nutritional status and a decreased survival rate, there still is a debate as whether the increased mortality rate is caused by malnutrition or by the advanced liver diseases itself [1]. This study aims to assess severity using prognostic scores and malnutrition among cirrhotic patients using a nutrition assessment tool, anthropometry and functional assessment and to assess association between severity of liver diseases and malnutrition. Malnutrition worsens the prognosis in liver cirrhosis, requiring early assessment of nutritional status of these patients [8]. Early diagnosis and treatment of malnutrition in cirrhotic patients is of great clinical importance and helps to identify the possible areas for nutritional intervention. If malnutrition of a cirrhotic patient is timely diagnosed, proper dietary counselling can be done and timely nutritional support can be provided to patient to avoid further depletion of nutritional status.

Early nutritional diagnosis and early intervention to improve nutritional status of the malnourished patient has favorable impact on prognosis which means it can reduce mortality of cirrhotic patients in waiting list for transplantation. The first and most important step in identifying patients with possible malnutrition is performing a thorough nutrition assessment using the most appropriate tools to evaluate their food intake and body composition, followed by proper nutrition intervention [8].

Functional assessment allows to study the quality of lean tissue (muscle strength) and it is useful for the global assessment of patient's nutritional status. Muscle weakness may insidiously develop with decompensation and malnutrition in patients with cirrhosis. Several studies documented an association between altered functional measures (e.g., handgrip, 6-minute walk test, physical frailty, and volume of oxygen peak tests) and bad prognosis $[9,10]$.

Previous study conducted in TUTH by Sherpa et al [5] where 50 patients were included and in Bir Hospital by Khadka et al [8], where 60 patients were included, had not included any information on functional assessment of admitted cirrhotic patients which could have been a major determinant in correlating it with malnutrition and prognostic scores. These studies conducted in Nepal have not included Hand Grip Dynamometer (HGD) for functional assessment which is a simple, inexpensive and sensitive tool for assessing the nutritional status in alcoholic liver disease and can reliably predict its complications and survival. This method has overall higher sensitivity and specificity with respect to other tools, in predicting sarcopenia [11]. Hand-Grip Strength (HGS) assessment method is relatively new measure of functional assessment in our context. Researches are needed for both dieticians and clinical practioners to determine which nutritional assessment tools to use in patients with liver diseases and whether different tools are needed for outpatients versus inpatients [12]. Information about nutritional status of cirrhotic patients in a tertiary care center provides an overview so that it helps in planning and prioritizing nutritional intervention to manage and reduce poor prognosis of patient. Multicenter studies using a common nutritional screening/assessment strategy are the next steps to fast-track adoption and implementation of nutrition-related evaluations into routine clinical practice.

The general objective of this study is to assess severity of liver cirrhosis and nutritional status of patients in Tribhuvan University Teaching Hospital, Kathmandu. 


\section{Material and methods}

A quantitative cross-sectional hospital-based study was conducted among the patients with liver cirrhosis admitted to the Gastroenterology department of Tribhuvan University Teaching Hospital. TUTH is specifically selected as study site because this study is a hospital-based study and TUTH is a tertiary care centre to manage patients with liver cirrhosis. Study was conducted over 6 months from November 2019 to April, 2020.

The study population were the patients with medically diagnosed case of liver cirrhosis admitted to the Gastroenterology department of TUTH of age group $\geq 18$ years. As this study is a hospital based, this study used the Non probability purposive sampling technique. Consecutive samples were collected unless and until the sample size was met. All the patients of 18 years or more who met inclusion criteria were the respondents of this study. The sample size was determined by using single proportion formula assuming the prevalence rate of liver cirrhosis to be $28.5 \%$ in the study area, 95\% confidence interval (CI), 10\% margin of error (d) [13]. The sample size is calculated to be 78.

When $10 \%$ non-response rate was added, total number of samples was 88.

Exclusion criteria included cirrhosis with coexisting tuberculosis, HIV or septicemia, coexisting hepatocellular carcinoma and other malignancies. Data was collected from February 11 to March 27, 2020 for 6 weeks. Data collections were carried out by using a proforma. Before data collection, patients' admission book was observed for collecting the information about diagnosis of the patient and selecting the medically diagnosed cases of Liver cirrhosis. Informed consent was taken from the patient explaining the objective of this research and the contribution of respondent to this research. Data on Demographic and general profile of patients and SGA were collected by doing face to face interview with the participants. Data regarding CTP score and MELD- Na scores were collected by observing the medical reports of patients. Data on Anthropometry and HGS were collected by using measuring tape, caliper and handgrip dynamometer.

Data collection tool, used in this study was a proforma which have following parts:

- Part 1: Demographic and general profile of patient

- Part 2: Liver Diseases Severity Scores: CTP-score, MELD-Na Score Standard Child Pugh Scoring was used. Standard MELD Na scoring system was used.

- Part 3: Nutritional Assessment Tool- Subjective Global Assessment (SGA) Subjective global assessment (SGA) according to the proposition of Detsky et al [14] was used.

- Part 4: Anthropometry- Mid Arm Circumference (MACcm), Triceps Skin Fold Thickness (TST cm), Mid Arm Muscle Circumference (MAMC cm)

- Measuring Tape for measuring MAC was standardized using stadiometer of UNICEF for children. Caliper used to measure TST was the one which was used in previous research [5].

- $\quad$ Part 5: Functional Assessment: Handgrip strength (HGS)

- $\quad$ Camry Handgrip Dynamometer was used to measure HGS [15].

All assessments were done by the principle investigator to avoid any inter observer variations.

Triceps skinfold thickness (TST) was measured by using skinfold caliper: Accu Measure, Item Model Number: 060379. A.R. Frisancho (1981) published tables showing percentiles for the thickness of the triceps skinfold [16]. Below P10, the patient was malnourished [17]. TST was categorized as normal: more than and equal to P10 and malnourished: less than P10 for analysis.

Mid-arm circumference (MAC) was measured by using non-stretchable measuring tape. Reference Percentile was taken from tables published by A.R. Frisancho [16]. MAC was categorized as normal: more than and equal to P10 and Malnourished: less than P10 for analysis.

Mid-arm muscle circumference (MAMC) was calculated by the following formula using

MAMC $=$ MAC $-($ TST $\times 0.3142)$. Reference for MAMC and TST was obtained from table by A.R Frisancho[16] . MAMC was categorized as normal: more than and equal to P10 and malnourished: less than P10 for analysis. 
A mechanical Hand Grip Dynamometer of size $10.5 * 15 \mathrm{~cm}$, Camry Hand Dynamometer Grip Power Strength Meter of $130 \mathrm{~kg} / 286$ lbs Capacity Force Gauge I8N was used to assess hand grip strength. Normal reference values for handgrip were taken according to age and sex matched data from Annals of Gastroenterology. All values were recorded in kg. HGS was categorized into weak, normal and strong following the reference value for handgrip strength given in Annals of Gastroenterology. For analysis, handgrip strength was categorized into impaired grip strength and non-impaired grip strength.

Three readings were noted with a gap of more than $30 \mathrm{sec}$ for all measurements. The mean of the 3 recordings were taken into consideration except for handgrip strength. For handgrip strength, second measurement value was noted.

\subsection{Severity of diseases}

CTP score: This scoring was done using format of CTP scoring [17]. Child Pugh's Score was classified as: A (5-6), B (7-9) and $\mathrm{C}(10-15)$.

MELD Na score: Model for End Stage Liver Diseases scoring was done by using preset format. This will be calculated by using following formula: [18]

Updated MELD $=0.957 \times$ Loge $($ creatine $\mathrm{mg} / \mathrm{dl})+0.378 \times$ Loge $($ bilirubin mg $/ \mathrm{dl})+1.120 \times$ Loge $($ INR $)+0.6431$

Updated MELD-Na = MELD + $1.32 \times(137-\mathrm{Na})-[0.033 \times \mathrm{MELD} \times(137-\mathrm{Na})]$

The maximum MELD score is 40. MELD- Na is a continuous score, the higher score the patient gets, the more severe is the liver cirrhosis and the patient is prioritized more for liver transplantation. For easy interpretation, MELD- Na score is classified into three categories [20]:

- $\quad$ Mild liver disease (score 6 - 15)

- Moderate liver disease (score 16 - 24)

- $\quad$ Severe liver disease (score $\geq 25$ )

For pretesting, a small pilot test was carried out in $10 \%$ of similar study population in the same hospital to assess the appropriateness and clarity of the questionnaire. Independent variables were TST, MAC, MAMC, HGS, SGA. Dependent variables were CTP- score, MELD- Na score. Association was tested between the following variables: CTP score with TST, CTP score with MAC, CTP score with MAMC, CTP with HGS, CTP with SGA, MELD- Na with TST, MELD- Na with MAC, MELD-Na with MAMC, MELD- Na with HGS and MELD-Na with SGA.

Data was then entered and analyzed by using Microsoft Excel- 2016 and SPSS version 20. The significance of study parameters on categorical scale between two or more groups was made by Chi-square to study the association between study variables. Test of significance was performed using independent sample t- test to compare means of different variables. One way ANOVA was used to study the relationship between continuous and categorical variable. All probability values less than $0.05(\mathrm{p}<0.05)$ were considered statistically significant.

For anthropometric measurements, three readings were noted with a gap of more than 30 sec. The mean of the 3 recordings was taken into consideration.

Ethical Approval was taken from Nepal Health Research Council (NHRC), a statutory and autonomous body under the government of Nepal. Written consent was obtained from all the literate participants while verbal consent was obtained from illiterate participants

\section{Results and discussion}

Out of 88 respondents; more than half were males. More than $3 / 4^{\text {th }}$ respondents belonged to the age group $\geq 35$. Mean age of all respondents was $54.82(\mathrm{SD}=10.75)$ years. Higher percentage of the respondents were Hindu accounting $3 / 4^{\text {th }}$ of the total number of respondents followed by Buddhist and the least belonging to others. More than half belonged to Janajati and the least belonged to others (11.4\%) but the percentage of respondents belonging to Brahmin and Chhetri were quite similar. Most of respondents were involved in agriculture. Regarding, personal habits, out of total respondents, most of the respondents had smoking and alcoholic habit. The most common etiology of the cirrhosis was found to be alcohol (77.3\%). 
More than half of the patients belonged to CTP class C (Decompensation of liver) and the least belonged to CPT Class A (Well-functioning liver). The mean MELD-Na score was found to be 19.67(SD=6.389) which means moderate liver diseases. Minimum MELD Na score was 9, meaning mild liver diseases and maximum MELD-Na score was 33 meaning severe liver diseases. The above information is shown in Figure 1

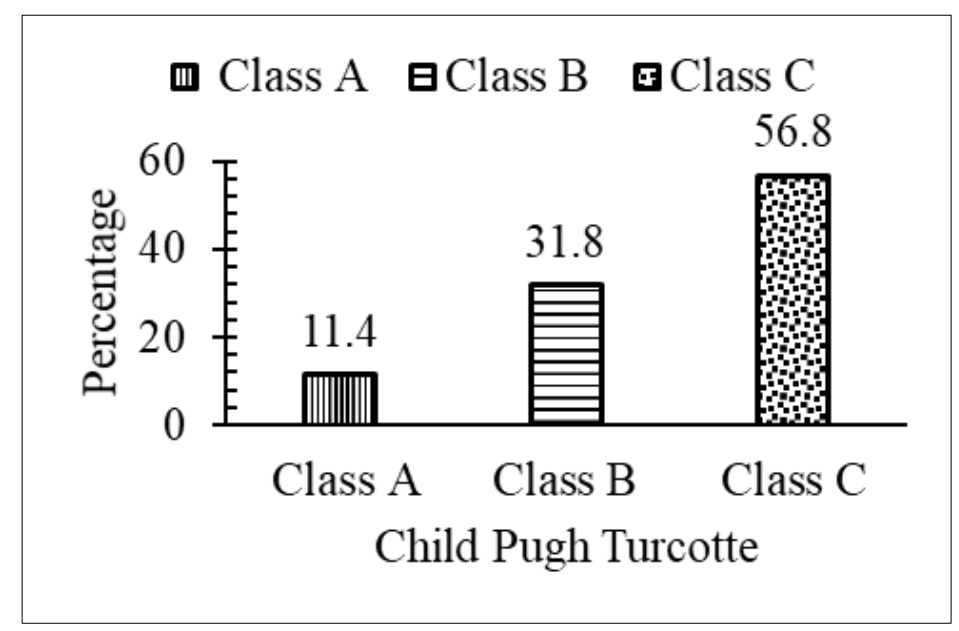

Figure 1 Severity of disease

Based on history and physical examination of the patient, patients were subjectively rated as well nourished (A), moderately malnourished (B), or severely malnourished (C). Out of total 88 respondents, the SGA classification showed that most of the respondents belonged to SGA class $\mathrm{C}$ as in given Figure 2.

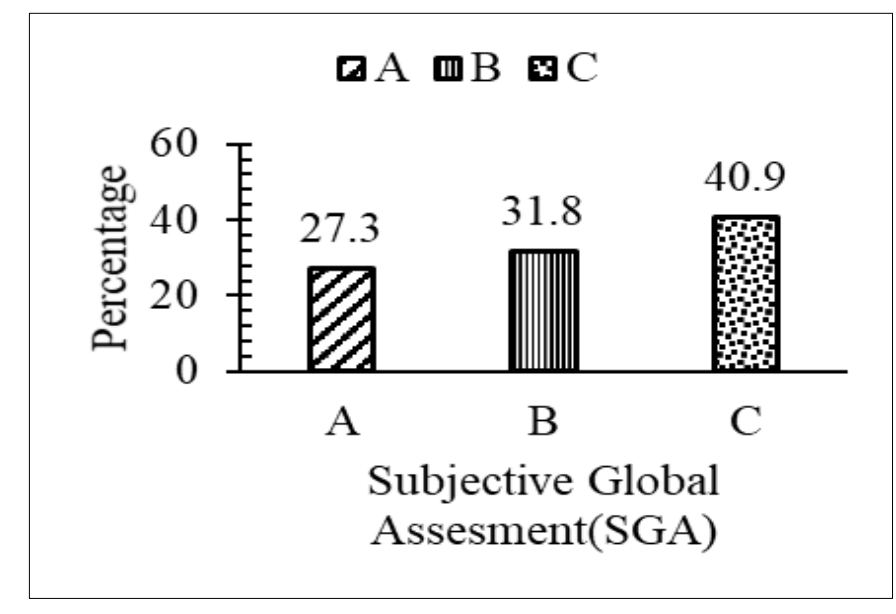

Figure 2 Nutritional status of cirrhotic patients by SGA

Both the TST and MAC showed that majority belonged to $<10$ th percentile and were malnourished. Furthermore, exactly half number of respondents were malnourished when assessed through MAMC. Based on the findings, TST and MAC were found to be more effective in determining malnourishment as compared to MAMC. This information is shown which is shown in Figure 3.

Present study revealed that out of 88 respondents, number of patients with impaired handgrip strength were about five times higher than the number of patients with non-impaired handgrip strength which is shown in Figure 4. 


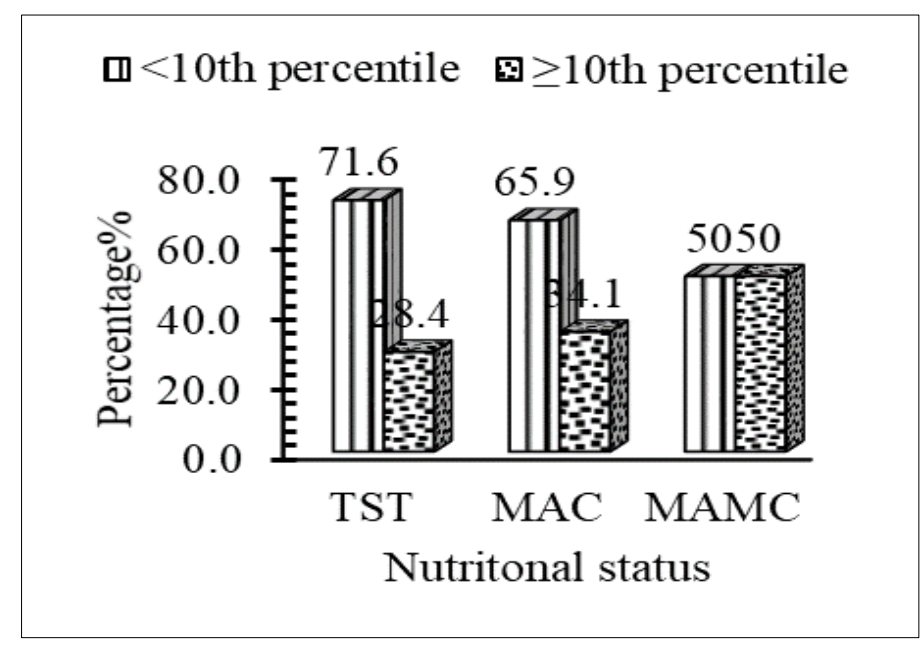

Figure 3 Nutritional status of cirrhotic patients by anthropometry

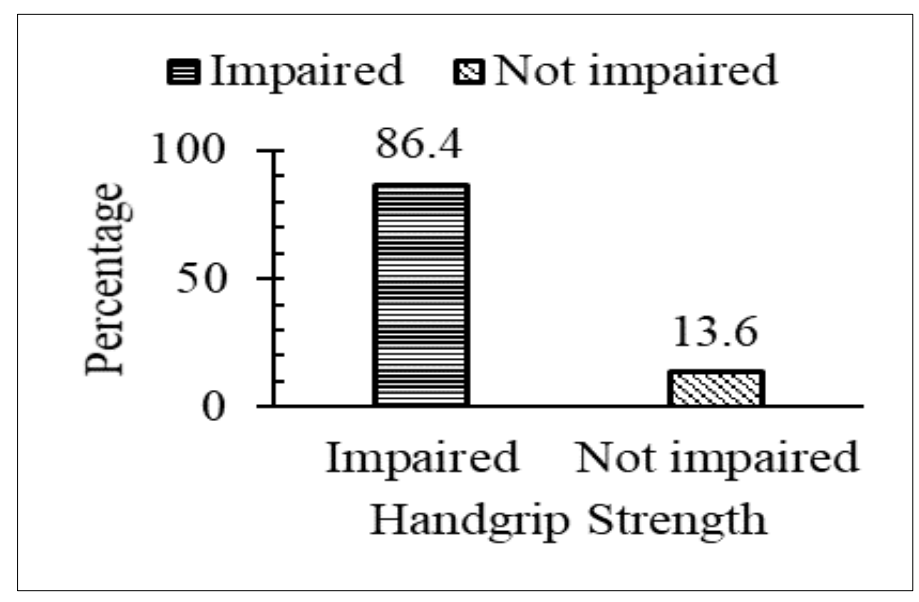

Figure 4 Handgrip strength of cirrhotic patients

Among 88 respondents, the number of respondents with decreased dietary intake was about 5 times higher than those with unchanged dietary intake. Concerning the type of diet that the patients were taking during hospitalization, about $3 / 4^{\text {th }}$ of the patients had been having suboptimal solid diet, about $1 / 3^{\text {rd }}$ of them were having minimal clear fluid and the rest had been having full fluid or any nutritive supplement. Usually cirrhotic patients require about 25 to $35 \mathrm{kcal} / \mathrm{kg}$ body weight depending upon their complications.

Out of total respondents, least number of patients had less than 5\% loss in their body weight during past 6 months, whereas half of the respondents had greater than $10 \%$ loss in body weight respectively. Although, the present study revealed that least number of patients had denied about the loss in functional capacity and majority had said that there was decrease in their functional capacity, only about $1 / 4^{\text {th }}$ of the total respondents were bed or chair ridden. About half of the respondents had difficulty with normal activity or ambulation.

\subsection{Association of disease severity with malnutrition and handgrip strength}

The present study showed that there was significant association between CPT score and SGA ( $p=0.004)$, CTP score and HGS ( $p=0.001)$, CTP score and TST $(p=0.006)$, CTP score and MAC $(p=0.001)$ but could not found any statistically significant association between CTP score and MAMC $(\mathrm{p}=0.115)$ which is shown in Table 1 . The study revealed that patients who belong to CTP class B and C were more malnourished when assessed using SGA, TST and MAC with impaired handgrip strength than those belonging to CTP class A. Whereas in this study, those patients belonging to CTP class B had equal number of normal and malnourished patients based on MAMC as a result no statistical association was seen between CTP and MAMC. 
Table 1 Association between CTP and TST, MAC, MAMC, SGA and HGS

\begin{tabular}{|c|c|c|c|c|}
\hline \multirow{2}{*}{ Variables } & \multicolumn{3}{|c|}{ Severity Index } & \multirow{2}{*}{ p value } \\
\hline & $\mathbf{A}$ & B & C & \\
\hline \multicolumn{5}{|l|}{ SGA } \\
\hline A & $6(25 \%)$ & $12(50 \%)$ & $6(25 \%)$ & \multirow{3}{*}{$0.004^{*}$} \\
\hline $\mathrm{B}$ & $2(7.1 \%)$ & $8(28.6 \%)$ & $18(64.3 \%)$ & \\
\hline $\mathrm{C}$ & $2(5.6 \%)$ & $8(22.2 \%)$ & $26(72.2 \%)$ & \\
\hline \multicolumn{5}{|l|}{ TST } \\
\hline$<10^{\text {th }}$ Percentile & $4(6.3 \%)$ & $17(27 \%$ & $42(66.7 \%)$ & \multirow{2}{*}{$0.006^{*}$} \\
\hline$\geq 10^{\text {thPercentile }}$ & $6(24.0 \%)$ & $11(44.0 \%)$ & $8(32.0 \%)$ & \\
\hline \multicolumn{5}{|l|}{ MAC } \\
\hline$<10^{\text {thPercentile }}$ & $2(3.4 \%)$ & $16(27.6 \%)$ & $40(69.0 \%)$ & \multirow{2}{*}{$0.001^{*}$} \\
\hline$\geq 10^{\text {thPercentile }}$ & $8(26.7 \%)$ & $2(40 \%)$ & $10(33.3 \%)$ & \\
\hline \multicolumn{5}{|l|}{ MAMC } \\
\hline$<10^{\text {thPercentile }}$ & $2(4.5 \%)$ & $14(31.8 \%)$ & $28(63.6 \%)$ & \multirow{2}{*}{0.115} \\
\hline$\geq 10^{\text {th }}$ Percentile & $8(18.2 \%)$ & $14(31.8 \%)$ & $22(50 \%)$ & \\
\hline \multicolumn{5}{|l|}{ HGS } \\
\hline Not Impaired & $6(50 \%)$ & $2(16.7 \%)$ & $4(33.3 \%)$ & \multirow{2}{*}{$0.004^{*}$} \\
\hline Impaired & $4(5.3 \%)$ & $26(34.2 \%)$ & $46(60.5 \%)$ & \\
\hline
\end{tabular}

This study revealed no any significant difference between the mean MELD Na scores among patients having TST, MAC and MAMC less than $10^{\text {th }}$ percentile and $\geq 10^{\text {th }}$ percentile $(p>0.05, p=0.158, p=0.078, p=0.703$ respectively) whereas showed significant difference between the mean MELD Na scores among patients having HGS Impaired and NonImpaired. $(P<0.05, p=0.045)$ and among patients belonging to different SGA class $(p<0.05, p=0.002)$ was found. This means as the MELD Na score increases in patients and severity of diseases increases, patient is more malnourished and has decreased handgrip strength whereas this study did not show any significant differences diseases severity due to TST, MAC and MAMC. The above information is shown in Table 2.

Table 2 Association of MELD Na with TST, MAC, MAMC, SGA and HGS

\begin{tabular}{|l|c|l|}
\hline Variable & \multicolumn{2}{|c|}{ MELD Na } \\
\hline & Mean \pm SD & p value \\
\hline SGA & $17.17 \pm 7.35$ & $\mathbf{0 . 0 0 2}^{*}$ \\
\hline A & $23 \pm 5.90$ & \\
\hline B & $18.75 \pm 4.99$ & \\
\hline C & & \\
\hline TST & $19.06 \pm 5.28$ & 0.158 \\
\hline$<10$ th percentile & $21.20 \pm 8.5$ & \\
\hline$\geq 10$ th percentile & & \\
\hline MAC & $20.53 \pm 5.15$ & 0.078 \\
\hline$<10$ th percentile &
\end{tabular}




\begin{tabular}{|l|c|c|}
\hline$\geq 10$ th percentile & $18 \pm 8.11$ & \\
\hline MAMC \\
\hline$<$ 10th percentile & $19.41 \pm 6.07$ & 0.703 \\
\hline$\geq 10$ th percentile & $19.93 \pm 6.75$ & \\
\hline HGS & $16.25 \pm 6.37$ & $\mathbf{0 . 0 4 5}^{*}$ \\
\hline Not Impaired & $20.21 \quad 6.26$ & \\
\hline Impaired & p value $<0.05^{*}$ \\
\hline
\end{tabular}

\section{Discussion}

Despite the fact that the present study population belonged to the age group above 18, as in all the previous literatures, more than $90 \%$ of the respondents belonged to the age group greater than 35 , late adulthood. The mean age of the study respondents was found to be $54.82(\mathrm{SD}=13.691)$ years. This is similar to the studies conducted in TUTH, Nepal in 2019 by Sherpa et. Al [5] and Uberlandia in 2013 by Viera et al [21], where the mean age was between 50 to 60 years. Study conducted in Pakistan by Naqvi et al [22] during 2009-2010, revealed that the mean age of the respondents was below 50.

The study showed that more than 50\% were Hindus and majority were from Hilly region. Mostly, respondents were from Janajati ethnic group. Most of the respondents had their occupation as agriculture. Similar results were observed in the previous study conducted in Nepal by Bhattarai et al [4]. Majority of the respondents had alcoholic and smoking history before hospitalization due to liver diseases.

The present study revealed that the most common aetiology for the liver cirrhosis was alcohol, followed by NASH. Regarding aetiology, similar result was also obtained from the studies conducted in TUTH by Sherpa et al [5] and Bir Hospital, Nepal by Khadka et al [8], where the sample size was 64 and 60 respectively[5,8]. The possible reason behind this aetiology in present study is majority of the participants belonged to Janajati. This can be justified by a finding of a cross-sectional descriptive study conducted by Maharjan et al in 2017 [23] at Bhaktapur among 250 samples where $56.8 \%$ belonged to Janajati which had shown that association of alcohol consumption was found to be highly significant with ethnicity and also stated that Brahmin and Chettri were 0.35 less likely to drink alcohol than other ethnic group [23]. To the contrary, another study conducted in Pakistan during 2010 by Naqvi et al [22] showed that the topmost aetiology of disease was Viral Hepatitis, alcohol being the rare cause. They have given explanation that the reason behind the topmost aetiology as Viral Hepatitis but not alcohol is due to the cultural barriers in that country due to religious prohibition of alcohol by Muslims.

Present study revealed that $88.6 \%$ of the patients had severe cases where $56.8 \%$ belonged to the CTP class $\mathrm{C}$ and $31.8 \%$ belonged to CTP class B. Only $11.4 \%$ of the study population belonged to CTP class A and had well-functioning liver. This result was similar to the result obtained from a study conducted in TUTH, Nepal by Sherpa et al [5], where 58\% of the patients belonged to CTP class C, followed by CTP class B with none of the patients belonging to CTP class A [5]. This was different from the result found in study conducted by Naqvi et al in Pakistan during 2009-2010 [22], Bangladesh by Rana et al during 2012-2013 [24] and Romania by Ciocirlan et al [25] during 2015-2016, where about half of the patients belonged to the CTP class B and the least belonging to the CTP class A with well-functioning liver. More severe disease in this present study as compared to other studies is probably due to study area, being a tertiary center, referred patients are likely to have severe disease than those presenting at other centers.

Present study showed that the mean MELD Na score of the study population was $19.67(\mathrm{SD}=6.38)$ indicating moderate liver disease. The minimum MELD Na score was 9 indicating mild liver diseases whereas the maximum score was 33 indicating severe liver diseases. This result was similar to the result obtained from the study conducted in TUTH by Khadka et al [8] where the mean MELD Na score was $19.64(\mathrm{SD}=6)$. Similar study conducted in Romania by Ciocirlan et al [25] during 2015-2016 showed the mean MELD Na score as $15.4(S D=6.5)$ which was slightly different from the result of the present study.

In this study, $40.9 \%$ belonged to SGA class C (severely malnourished) followed by $31.8 \%$ SGA class B (moderately malnourished) and $27.3 \%$ SGA class A (normal) patients. This result was similar to the result obtained from the study conducted in Bir hospital, Nepal by Khadka et al [8], where majority of the patients belonged to SGA class C followed by 
the number of patients belonging to the SGA class B and A. Majority (72.7\%) belonged to the malnourished group(SGA B or C) in present study which is higher than the reported, 66\% malnourished (SGA B or C), in Romanian study by Ciocirlan et al [25]. This difference may be partly explained by the fact that we included patients with more severe disease (present study-11.4\% Child-Pugh A, 31.8\% Child-Pugh B, and 56.8\% Child C and Romania study-23\% ChildPugh A, 46\% Child-Pugh B, and 31\% Child-Pugh C). The present study revealed that alcohol is the top aetiology which is supported by previous studies by Campillo et al [26] and Fernandes et al [27]. Both studies demonstrated a higher incidence of malnutrition in alcoholic cirrhotic compared to other aetiologies of cirrhosis, and these patients had more complications of cirrhosis.

Majority of the patients $(71.6 \%)$ had TST less than $10^{\text {th }}$ percentile and remaining $28.4 \%$ had TST $\geq 10^{\text {th }}$ percentile. This result was different from the similar study conducted in TUTH, Nepal by Sherpa et al [5], Pakistan by Naqvi et al [22] and Bangladesh by Rana et al [24], where the patients falling into the malnourished category were $52 \%, 45.97 \%$ and $39 \%$ respectively which is far less than the present study. It might be because those studies had used lower cut off level (less than or equal to $5^{\text {th }}$ percentile) to define malnutrition according to TST which might have underestimated the actual result. Although it is theoretically possible that the presence of swelling could hide a depletion in adipose tissue and that the frequency of diagnosis of malnutrition could be reduced by the TST method, it has been reported that in patients with chronic liver disease, the upper limbs are not the preferential place of swelling, principally in the case of patients who are not confined to bed [28]. But in this present study, only $22.7 \%$ of the patients were bed or chair ridden despite the fact majority of the patients had decreased functional capacity. Furthermore, study conducted among 101 patients in Egypt during 2015-2016 by Hassan et al [20] showed 47.2\% had TST $<10^{\text {th }}$ percentile, which is lower than $71.6 \%$, observed in present study. This might be because $88.6 \%$ had severe liver diseases in present study which is higher than $78.2 \%$ severe cases observed in study by Hassan et al [20].

In this study, majority (65.9\%) of the patients had MAC less than $10^{\text {th }}$ percentile and only $34.1 \%$ had MAC more than or equal to $10^{\text {th }}$ percentile. This result was lower from the result obtained from the study conducted by Hassan et al [20] among 101 patients during 2015-2016 in Egypt where 75.2\% had lower MAC. A study conducted by Fernandes et al [27] revealed that $14 \%$ cirrhotic patients had lower MAC which is quite lower than the observed, in present study which might be because of majority of the respondents belonging to CTP class B and C. Similarly, exactly half of the total study population had MAMC less than $10^{\text {th }}$ percentile which is very high than $13.2 \%$ observed in the study by Fernandes et al [27], which might be because of the fact that, $51 \%$ of the respondents in our study had observed more than $10 \%$ of their body weight loss in the past 6 months and majority had severe liver diseases belonging to CTP class B and C. Study conducted in Egypt by Hassan et al [20] during 2015-2016 had stated that $67.3 \%$ of the patients had MAMC below $10^{\text {th }}$ percentile which is still higher than that have been obtained in present study. The greater frequency of diagnosis of malnutrition by TST than by MAMC could be associated with metabolic alterations arising from liver cirrhosis. In patients with chronic hepatic insufficiency, lipids are used as a preferential metabolic substrate for energy production [28]. Besides this, individual anthropometrical variations are not considered in the calculation of MAMC. It is taken for granted that the humerus has the same diameter in all groups of the population and that the arm has a cylindrical instead of an elliptical form [29].

Based on SGA, majority of the patients (84.1\%) had stated that they have decreased dietary intake. This is due to various symptoms due to diseases and complications affecting oral intake like pain while eating, anorexia, vomiting, nausea, dysphagia, diarrhea, dental problems, feeling of early satiety, constipation. Also, hospitalized patients have usually mild to severe diseases symptoms. Only $15.9 \%$ had stated that their dietary intake remained unchanged. Majority of the patients $(70.5 \%)$ were reported to have been eating suboptimum solid diet, $23.9 \%$ of the patients were under minimum clear fluid and $5.7 \%$ of them were having full fluid or nutritive supplements only.

Majority of the patients had said that their functional capacity had decreased (73.9\%). Out of total respondents, 53.4\% had said that they had difficulty with ambulation or normal activities. $22.7 \%$ were chair or bed ridden. $86.4 \%$ of the patients had impaired handgrip strength indicating majority of the patients had sarcopenia. Muscle wasting in the cirrhotic is an indirect evidence of the malnutrition. This muscle wasting correlating with the functional loss can be measured with the Handgrip strength (HGS). In a study by Abbott et al, it was found that reduced HGS was associated with decreased physical capability [30].

This present study revealed statistically significant association of SGA with CTP class and MELD Na which is similar to the results obtained from study conducted in Romania by Ciocirlan et al [25] where SGA significantly correlated with Child-Pugh, and MELD-Na scores. Present study revealed that patients belong to SGA class B and C with increasing cirrhosis severity as estimated by Child-Pugh class and MELD-Na scores. In relating the nutritional state of the patients to the severity of the hepatic dysfunction, a greater number of moderately malnourished and of severely malnourished patients was observed by SGA, respectively, in Child-Pugh B $(8 / 28 ; 8 / 36)$ and C $(18 / 28,26 / 36)$ than in A (2/28 and 
2/36) and majority of the respondents (88.6\%) belonged to CTP class B or C. Similarly, mean MELD Na scores were found to be higher in patients belonging to SGA class B and C in comparison to that of SGA class A. Whereas, there was no significant variation in SGA classes between groups of MELD Na scores in a study conducted in Egypt by Hassan et al [20].

The present study revealed that there was statistically significant association between CTP and TST and MAC suggesting that lower the values of TST and MAC, worse is the diseases severity but didn't show association with MAMC. It was found that the number of malnourished patients were greater $(\mathrm{p}<0.05)$ among those classified as Child-Pugh C (TST: 42/63) and MAC: 40/58) than among those classified as Child-Pugh A (TST: 4/63) and MAC (2/58). The study was conducted in TUTH by Sherpa et al [5] which also did not show any significant statistical association between MAMC and CTP class. Similar study conducted in Romania whose aim was to evaluate malnutrition assessment tools in predicting severity and survival of patients with liver cirrhosis where MAMC (which is derived from MAC and TST) was used and did not find any correlation between MAMC and the severity of liver. That study had provided explanation that $31 \%$ of their patients were Child-Pugh $\mathrm{C}$ and $75 \%$ had ascites. To the support of this information, present study showed that majority, $88.6 \%$ of the patients belonged to CTP class B or C and $73.9 \%$ of the patients had fluid overload. Fluid overload with ascites and peripheral oedema was more frequent in their study group which can explain overestimation of nutritional status by MAMC and lack of correlation with disease severity. In advanced stages, MAMC will suffer changes, but it may also be overestimated due to fluid retention, hence its lack of correlation with liver disease severity.

Similarly, there was no any statistically significant difference between the means of MELD Na among TST classes, MAC classes and MAMC classes indicating that no significant decrease in TST, MAC and MAMC in cirrhotic patient as the disease severity progresses with increase in MELD Na scores. This was similar to the Romanian study where there was no any statistical correlation between MAMC and MELD Na scores. Similarly, the result from the Egyptian study found that TST, MAC and MAMC were not showing significant decrease as the disease severity progressed, and that was consistent with what was established whereas no significant variation in the anthropometric measurements with the MELD Na score was found.

Study which was conducted with 60 patients with liver cirrhosis of different aetiologies treated in hepatology department in Ain Shams University hospitals and Wady El-Nile Hospital, during 2012-2013 by Monsef et al (2014) indicated that unlike the Child-Pugh score, the MELD Na score is not associated with the prevalence of malnutrition in regard to the severity of liver disease. In that study, only albumin and TLC were associated with MELD Na which indicates that this parameter is related to the degree of liver failure rather than to malnutrition. We might thus assume that those who are transplanted first are not necessarily the more severely malnourished. On the other hand, mortality while on the waiting list might be associated with malnutrition, the longer they remain on it. However, to confirm this theory, further studies are needed.

According to the present study, there was statistically significant association between CPT and HGS and, MELD Na and HGS. This result is supported by the study conducted in Romania by Ciocirlan et al [25] in 2015-2016, where HGS significantly correlated with cirrhosis prognostic scores (CPT and MELD Na). In contrary to these results, a study by Fernandes et al [27] showed no correlation between HGS and Child-Pugh classification where patients with HCV (43.4\%) and alcoholic aetiology (25.6\%) were included and mostly patients were with compensated liver cirrhosis (91 of 129 patients were Child-Pugh A, 27 were Child-Pugh B, and only 9 were Child-Pugh C). Furthermore, they tested the non-dominant hand and showed that HGS did not significantly decrease with increasing severity of liver cirrhosis (24.7 \pm 11.2 in Child-Pugh A, $26.6 \pm 14.3$ in Child-Pugh B, and $21.3 \pm 11.7$ in Child-Pugh C, $p=0.510$ ) whereas HGS of the dominant hand was tested in present study as done in study by Gaikwad et al [15]. Monsef et. al (2014) showed no association between HGS and MELD scores. The authors imply that dynamometry can lead to increased numbers of false positive diagnoses. In this present study the MELD Na values reported herein represent the values obtained in one given moment at the nutritional evaluation which might have influenced in the association.

In this study, nutritional indicators showed higher correlation with the Child-Pugh criteria than with MELD- Na. It is noteworthy that, in the previous version of the Child-Pugh criteria, Malnutrition level was considered as an indicator; however, the absence of an accurate tool for nutritional diagnosis has led to its exclusion. On the other hand, MELD estimation, so far, does not take nutritional status into account.

\section{Conclusion}

This study presented that the top common aetiology of liver cirrhosis was Alcoholic Liver Diseases where majority of the respondents belonged to the Child Pugh class $\mathrm{C}$ indicating decompensation of liver. The mean MELD Na score of the respondents was found to be $19.63 \pm 6.38$ indicating moderate liver diseases which is higher than the normal MELD Na 
scores, 6-15, which indicates mild liver diseases. Lower the MELD Na scores less is the degree of diseases severity. The present study revealed that $3 / 4^{\text {th }}$ of the respondents were malnourished when assessed using SGA and TST. More than half of the patients had lower MAC and half of the patients had lower MAMC. More than 3/4th of the respondents had impaired handgrip strength.

This study demonstrated significantly higher prevalence of malnutrition in higher CTP grades and MELD Na scores. Association between CTP and TST, MAC, HGS and SGA was found, indicating higher number of malnourished patients belonging SGA class B and C having decreased TST, MAC and HGS with increased disease severity. Whereas no any statistically significant association was observed among CTP and MAMC indicating, MAMC does not necessarily decrease with increase in diseases severity. Furthermore, MAC and MAMC were not found to be decreasing with increase in MELD Na scores showing no association with MELD Na scores. Whereas HGS was found to be decreasing with increase in MELD Na scores. Patients belonging to SGA class B and C were found to be having more MELD sodium scores indicating higher degree of diseases severity. The nutritional status of cirrhotic patients is an important tool, together with Child score and MELD score, for the prediction of prognosis of such patients, and the severity of malnutrition is associated with higher malnutrition.

Early diagnosis of the nutritional state of cirrhotic patients should be done which can contribute to a reduction in the frequency and severity of liver diseases. Cirrhotic patients with high degree of disease severity and poor nutritional status should be referred for early dietician consultation in order to provide early nutritional support. SGA, TST, MAC and HGS are the non-invasive and easy methods of nutritional assessment of cirrhotic patients to use in regular clinical practice at bed side.

Since this study was a single centred cross-sectional study, further multicentre studies with a large number of patients and comparable control group to see the prevalence of severe malnutrition with associated factors in cirrhotic patients is important. A national reference database of anthropometric measurements of the population needs to be developed to assess malnutrition with more accuracy. Regular assessment of nutritive intake and nutritional status is mandatory, better using anthropometric measurements such as the SGA, TST, MAC and Hand grip dynamometer.

\section{Compliance with ethical standards}

\section{Acknowledgments}

The authors would like to thank Miss Nani Shova Shakya and Mr Milan Dhakal for their help and support.

\section{Disclosure of conflict of interest}

The authors declare that they have no conflict of interest.

\section{Statement of informed consent}

Ethical Approval was taken from Nepal Health Research Council (NHRC), a statutory and autonomous body under the government of Nepal. Written consent was obtained from all the literate participants while verbal consent was obtained from illiterate participants.

\section{References}

[1] Saunders J, Brian A, Wright M, Stroud M. Malnutrition and nutrition support in patients with liver disease. Frontline Gastroenterol. 2010; 1(2): 105-111.

[2] Merli M, Riggio 0, Dally L. Does malnutrition affect survival in cirrhosis? PINC (Policentrica Italiana Nutrizione Cirrosi). Hepatology. 1996; 23(5): 1041-1046.

[3] National Institute for Health and Clinical Excellence, Nutrition Support in adults. NICE. 2006.

[4] Bhattarai S, Gyawali M, Dewan KR, Shrestha G. Demographic and Clinical Profile in Patients with Liver Cirrhosis in a Tertiary Care Hospital in Central Nepal. JNMA J Nepal Med Assoc. 2017; 56(208): 401-406.

[5] Sherpa T, Pathak R, Khadga P, Sharma S, Hamal R, Jha A. Nutritional Assessment of Patients with Liver Cirrhosis by Nutrition Screening Tool and Anthropometry at a Tertiary Care Center. J. of Ins. of Medicine Nepal. 2019; 41(2): 21-25. 
[6] Amodio P, Bemeur C, Butterworth R, et al. The nutritional management of hepatic encephalopathy in patients with cirrhosis: International Society for Hepatic Encephalopathy and Nitrogen Metabolism Consensus. Hepatology. 2013; 58(1): 325-336.

[7] Eghtesad S, Poustchi H, Malekzadeh R. Malnutrition in liver cirrhosis: the influence of protein and sodium. Middle East J Dig Dis. 2013; 5(2): 65-75.

[8] Khadka D, Karki B, Thapa S, et al. Prevalence of Malnutrition in Patients with Liver Cirrhosis in A Tertiary Care Hospital. JNMA J Nepal Med Assoc. 2019; 57(218): 229-233.

[9] Alameri HF, Sanai FM, Al Dukhayil M, et al. Six Minute Walk Test to assess functional capacity in chronic liver disease patients. World J Gastroenterol. 2007; 13(29): 3996-4001.

[10] Tandon P, Low G, Mourtzakis M, et al. A Model to Identify Sarcopenia in Patients with Cirrhosis. Clin Gastroenterol Hepatol. 2016; 14(10): 1473-1480.e3.

[11] Huisman EJ, Trip EJ, Siersema PD, van Hoek B, van Erpecum KJ. Protein energy malnutrition predicts complications in liver cirrhosis. Eur J Gastroenterol Hepatol. 2011; 23(11): 982-989.

[12] Patton HM. Nutritional assessment of patients with chronic liver disease. Gastroenterol Hepatol (N Y). 2012; 8(10): 687-690.

[13] Mishra A, Shrestha P, Bista N, Bhurtel P, Bhattarai S, Thakali K, Pathak S. Pattern of Liver Diseases. J Nepal Health Res Council [Internet]. 7 Oct 2009.

[14] Detsky AS, McLaughlin JR, Baker JP, Johnston N, Whittaker S, Mendelson RA, Jeejeebhoy KN. What is subjective global assessment of nutritional status? JPEN. J. of parenteral and enteral nutr. 1987; 11(1): 8-13.

[15] Gaikwad NR, Gupta SJ, Samarth AR, Sankalecha TH. Handgrip dynamometry: a surrogate marker of malnutrition to predict the prognosis in alcoholic liver disease. Ann Gastroenterol. 2016; 29(4): 509-514.

[16] Frisancho AR. New norms of upper limb fat and muscle areas for assessment of nutritional status. Am J ClinNutr. 1981; 34(11): 2540-2545.

[17] Pugh RN, Murray-Lyon IM, Dawson JL, Pietroni MC, Williams R. Transection of the oesophagus for bleeding oesophageal varices. The Br. J. of surgery. 1973; 60(8): 646-649.

[18] Blackburn GL, Harvey KB. Nutritional assessment as a routine in clinical medicine. Postgraduate med. 1982; 71(5): 46-63.

[19] Showkat, AM, Qayoom RA, Shabeena, P \& Raja, W. Prognostic Evaluation Of Chronic Liver Disease Patients With Various Scoring Systems. Int. J. of Current Res. And Rev. 2017; 17-21.

[20] Hassan MS, Abdel RAS, Eldin KMA, Mahmoud, OYA. Nutritional assessment of cirrhotic patients with variable severity. J. of Current Med. Res. and Practice. 2019; 4(2): 144-151.

[21] Vieira PM, De-Souza DA, Oliveira LC. Nutritional assessment in hepatic cirrhosis; clinical, anthropometric, biochemical and hematological parameters. Nutr Hosp. 2013; 28(5): 1615-1621.

[22] Naqvi IH, Mahmood K, Salekeen S, Akhter ST. Determining the frequency and severity of malnutrition and correlating it with the severity of liver cirrhosis. The Turkish Journal of Gastroenterology: the Official J. of Turk. Soc. of Gastroenterology. 2013; 24(5): 415-422.

[23] Maharjan P,Magar KT. Prevalence of Alcohol Consumption and Factors Associated with the Alcohol Use among the Youth of Suryabinayak Municipality, Bhaktapur. J. of Pharmaceutical Care \& Health Systems. 2017; 04.

[24] Rana M, Faisal MA, Karim ME, Siddique AR, Ahmed D, Raihan A. Assessment of Malnutrition in Cirrhotic Patients. Bangladesh J. of Medical Sc. 2016; 15(2): 189-194.

[25] Ciocîrlan M, Cazan AR, Barbu M, Mănuc M, Diculescu M, Ciocîrlan M. Subjective Global Assessment and Handgrip Strength as Predictive Factors in Patients with Liver Cirrhosis. Gastroenterol Res Pract. 2017; 8348390.

[26] Campillo B, Richardet JP, Scherman E, Bories PN. Evaluation of nutritional practice in hospitalized cirrhotic patients: results of a prospective study. Nutrition. 2003; 19(6): 515-521.

[27] Fernandes SA, Bassani L, Nunes F F, Aydos ME, Alves AV, Marroni CA. Nutritional assessment in patients with cirrhosis. Arquivos de gastro enterologia. 2012; 49(1): 19-27.

[28] Nompleggi DJ, Bonkovsky H. Nutritional supplementation in chronic liver disease: an analytical review. Hepatology (Baltimore, Md.). 1994; 19(2): 518-533. 
[29] Müller MJ, Böttcher J, Selberg O, et al. Hypermetabolism in clinically stable patients with liver cirrhosis. Am J Clin Nutr. 1999; 69(6): 1194-1201.

[30] Abbott WJ, Thomson A, Steadman C, Gatton ML, Bothwell C, Kerlin P, Wall DR, Lynch SV. Child-Pugh class, nutritional indicators and early liver transplant outcomes. Hepato-gastroenter. 2001; 48(39): 823-827.

\section{Author's Short Biography}

\begin{tabular}{|l|l|} 
Ms Yogita Sapkota is a practicing Dietician Supervisor of National Trauma Center, \\
National Academy of Medical Sciences. She has done her BND (Bsc Nutrition and Dietetics) \\
from Tribhuvan University and MND degree (Msc Nutrition and Dietetics) from \\
Purbanchal University. She has about 6 years of working experience in the field of \\
Nutrition and Dietetics.
\end{tabular}

